\title{
Towards a methodology for estimating the global impacts of innovative design scenarii
}

Dominique Deneux, Olivier Sénéchal, Frédéric Tomala, and Massi Lawson LAMIH - UMR CNRS $n^{\circ} 8530$ - University of Valenciennes - France

\begin{abstract}
This paper introduces a methodology aiming at reducing the risk of misestimating the future cost and value parameters of an innovation, so as to provide the managers with the instruments of a vision closer to the reality of the global impact. The goal of that method is to create representations of the analysed situation, useful for the decision to innovate, but also to foster the emergence of reusable knowledge for similar situations. So, we concentrate on the re-usable models of the product at design time, and on the relations that exist between the innovative "features", and the various processes.
\end{abstract}

Key words: Methodology, cost, value, global impact, life-cycle, innovation, design.

\section{INTRODUCTION}

According to (Xuereb 1991), the industrial companies must develop and manage their ability to innovate to remain competitive. But the implementation of a real "innovation engineering" requires the development of new models, methods and tools taking into account the specificity of this problem (Renaud et al. 1999), and cannot rely on those existing for traditional design. Today's manufacturing companies require agility to bear the external evolutions impacting their business, products or processes. The agility of a company is characterised by its ability to modify its products or processes within a short period of time and at a minimal cost. Actually, the need to innovate is generally due to external causes, such as the new offers from competitors, the consumers' changing requirements, the laws (environment, security standards, ...), the new technologies available for 
product (technology of materials, integrated electronics, etc), or new technologies available for the processes (information technologies, new manufacturing and production systems, ...). But innovation has a cost, and the companies must evaluate the expected impact of innovation upon the cost, correlatively with the value induced by this innovation. The main difficulty stems from the fact that, in a concurrent engineering situation, the cost, like the value, are shared by several actors. The success of an innovation is thus dependant on a sensible balance between the cost, shared by all cost supporters, and the added value, also shared by all the actors.

In the following we give a state of the art about innovation, so as to position our work. Then we analyse the requirements in terms of models, before to introduce our models.

\section{INNOVATION}

Schumpeter (Schumpeter 1935) was the first to insist on the necessary distinction between invention and innovation. Invention refers to the scientific and technical domains; it is a continuous process, which does not follow economical rules. Conversely, innovation refers to the economical domain, and is a discreet process. It is qualified in (Renaud et al. 1999) as a "value producing process". The value is a judgement made by the customer upon the product, based on his expectations and motivations, expressed by a variable, which increases when his satisfaction is increased, and when the related expense decreases. (NFX50-150 1983).

A typology of innovation was proposed in (Barreyre 1981), based on three criteria:

1. the intensity of innovation, according to pre-identified classes such as "classical", "just improved", "perfected", "new".

2. the intrinsic nature of innovation

- technical: new material, or products, or components, or processes, ...

- commercial: new distribution mode, or packaging, ...

- organisational: new project, or team work, or partnership, ...

- socio-institutional : new law or standard, ...

3. the characteristics of the innovation process

- triggering mode: response to a lack or a threat, reactive, proactive, ...

- management mode: intuition, improvisation, plan, program

In any project, there exist a risk. The notion of risk is defined as the possibility for the project, not to be achieved exactly as planned, considering the criteria of cost, time, or results (AFITEP, 1996). The risk is characterised by the severity of the feared event and by the probability of occurrence of 
that event. It is possible to characterise both parameters formally (Marciniak et al. 1999), but measuring the risk with indicators is not enough; it is also necessary to have some possibilities to influence these indicators. For this reason, it is necessary gather information about the innovation environment, which is composed of all elements constraining it.

The innovation environment can be limited to immediate elements such as the need and the market. According to (Fernez-Walch, 1991), the innovative product is a combination of a concept, a set of functions, and a set of resources, in relation with an uncertain environment.

The innovation process is the process by which an invention becomes an innovation. Several models can be used to represent the innovation process. The most basic one is the linear and sequential model, made of a succession of phases, from inventions to sales, separated by reviews (stop or continue). During the IMPLANT AIT-project, (Eschenbächer et al. 1999) a sequential model has been proposed to describe a generic enterprise change management methodology, permitting to evolve from an AS-IS situation to a TO-BE situation (see figure 1).

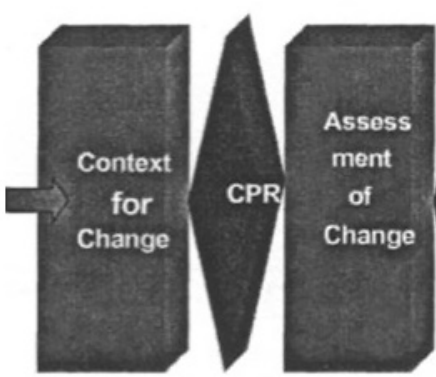

Decision to innovate

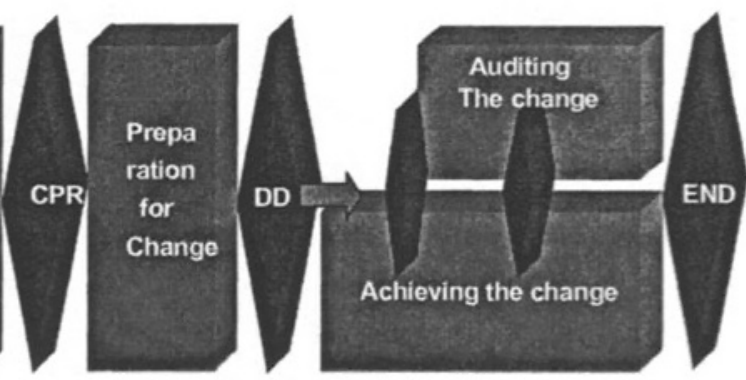

Decision to deploy

Figure 1.

In the process presented above, the generic path includes five principal activities, separated by change project reviews:

1. context for change : model the AS-IS situation of the enterprise, before the innovation,

2. assessment of change: estimating the consequences of the innovation, based on the model of the TO-BE situation,

3. preparation for change: develop an operational process for integrating the innovation into the enterprise,

4. achieving the change : integration of the innovation 
5. auditing the change: evaluate the difference between what was planned and what is obtained.

The model proposed in (Kline et al. 1986) includes short and long retroaction loops between all phases, from invention to marketing, and a global loop showing the continuous nature of the process. Moreover, links exist between the central spine of the process, and the academic world continuously producing inventions.

Methods and tools for supporting this process are necessary. Basically, we can distinguish those that are commonly used to support the design activity, from those specifically developed for supporting the innovation process.

Traditional tools and methods available to support the design activity can be classified into seven categories (Vadcard 1995).

1. Multidisciplinary description of the need: QFD, FAST, APTE, ...

2. Solution identification: brainstorming, matrix of discoveries, ...

3. Product definition

4. Solution representation: traditional $\mathrm{CAD}$ systems, geometric modellers, ...

5. Solution analysis: simulation tools, value analysis, failure modes analysis, ..

6. Project management: Gantt, PERT, MPM, ...

7. Quality oriented: cause effect, pareto, taguchi, ...

Tools and methods specifically designed to support an innovative process fall into two categories:

1. Dedicated to support the innovation strategy (Meredith et al. 1995) such as the technological survey, ...

2. Dedicated to support the operational aspects of innovation: Computer Assisted Innovation. The assistance may concern the process of innovation, like proposed by the IMPLANT electronic assistant, (Eschenbächer et al. 1999) or the emergence of an innovation, like in the TRIZ method (Nordlund et al. 1996).

Based on the survey presented above, we can now better characterise the objectives of our current work.

\section{INNOVATION CHARACTERISATION REQUIREMENTS}

We regard as innovation every existing product into which it has been determined useful to integrate a new technology, an invention, or new services. So, the innovation is essentially technical. The existing product can 
be considered as the situation of reference (AS IS). Consequently we do not consider radical innovations, these cases not having a situation of reference, but rather incremental innovations.

The generic actors to be taken into account can belong to any mobilisation space suggested by Gaillard (Gaillard, 1999). Since the innovation concerns the product functions, the customer is perceived as a key actor, as far as the value is concerned. However, it should be kept in mind that every actor has his own opinion of the value of the product, which is not dependant on other actor's points of view. The other actors to be considered are, for example, the manufacturer, the service provider, the cocontractor, ...

The precise problem which we address is the definition of a formal and structured framework for the modelling of the existing product (AS IS) on the one hand, and the characterisation of one or more alternatives (TO-BE) of the innovation on the other hand. Although we do not consider the problem of making a decision, we wish to facilitate the comparative study by the innovation project managers at least, and more generally by all the actors who are likely to intervene throughout the life cycle of the new product. This objective corresponds to the second phase of the IMPLANT innovation process management method (assessment of change).

Our goal is to support this phase, by means of design method and models adapted for the purpose of dealing with the kind of innovative design described above.

In the following, we firstly introduce the proposed method, then we detail the suggested models.

\section{INNOVATION CHARACTERISATION METHOD}

The method which we propose includes three distinct phases, that can (but need not to) be executed in a sequence, an entry point (input) which initiates the method but is generated externally, and an output which can be used outside the scope of the method, for example for taking a strategic decision concerning the innovation (the decision making process is not addressed in this paper). The different steps of the method are detailed in the following sections.

\subsection{Input}

The input is a prerequisite for the method. At this point, a target of innovation was identified and all useful information on the invention to be 
integrated was collected. The target determines the perimeter of the innovation effect. Along with the target, the objectives of performance for the innovation are also identified. The information can concern the available patents, the available technology providers, the technological status of the competition, the forecast of the market analysis, the other applications which already use this invention.

\subsection{Determine the actors}

Based on the previous identification of the perimeter of the innovation effect (which functions ?, which components ?, ..) it is possible to determine the actors of the various mobilisation spaces that are directly concerned with that innovation, i.e., those whose value indicators are modified by the innovation, or those who have to support a share of the cost related to the innovation. The actors whose value indicators are modified are the receptors of the new services or functions (basically, the customer) or the actors whose commercial activity is impacted by the introduction of the innovation (provider of impacted components, maintainer of these components, ...). All the actors concerned in any phase of the life-cycle of the product should be considered. Immediate actors can be identified in the first loop, and other actors can be revealed at a later loop. However, since all the actors are not equally impacted, a particular attention should be directed to the most concerned ones.

\subsection{Determine the AS-IS}

To each actor corresponds one or more specific models of representation. Some models of the AS-IS already exist within the company, if the innovation concerns a product that was designed by the company. If so, the models useful for the study can be identified. When the models do not exist in the company (because the AS-IS which is the reference of the market is a competitor's product), then the useful models are not only determined but rebuilt. The process is the same for all the actors identified in the previous phase. In order to support the consistence of the analysis, the use of common representation models is to be preferred.

\subsection{Characterisation of TO-BE versus AS-IS}

Starting from the models identified for representing the AS-IS, the specific features of the TO-BE can be represented in the form of a modification (edition, addition, suppression). The modifications can concern 
the data (e.g.: a value characterising the performance of a service function) or the variables (e.g.: the precision, the reliability, ...) describing the AS-IS. The modifications can then be propagated to the derived models, characterising the underlying levels of details.

\subsection{Output}

The output of the method should be in a form exploitable for the innovation project leader. It should represent the consequences for the cost and the perceived value, of the adoption of a candidate TO-BE, for all the concerned actors throughout the products life cycle, and comparatively to the AS-IS. So as to reach this objective, the key actors should directly (project reviews) or indirectly (questionnaires) deliver their opinion about the criticality of each specific feature of the TO-BE.

Models are necessary to support this method. The following section describes an early version of the models we are currently working on.

\section{INNOVATION CHARACTERISATION MODELS}

L. Jacquet (Jacquet 1998) proposed a modelling framework to support a functional design activity. This framework is organised in five modelling domains, specifically dedicated to represent the need (what the customers wants), the functional requirements of the need (technical functions), the technological aspects, (associated to symbolic solutions), the technical aspects, (physical solutions) and the detailed aspects of the design. In the first domain (the need) Jacquet suggested the use of three representation models, inspired from the value analysis and QFD methods: the octopus, the external elements characterisation matrix, and the specification matrix.

The models proposed by Jacquet in the first modelling domain proved to be useful to represent and characterise the need addressed by the innovation. So they were adapted for representing the innovation in a comparative manner (TO BE vs AS-IS). These models are recalled in the following sections, as well as the criticality analysis matrix, which is a new model.

\subsection{The octopus}

The octopus model is used to graphically represent the system to be designed and the relation he has with the influent external elements (existing outside the system). Each relation between two elements realised thanks to the system determines a service function (SF). 
This model, suggested by the AFAV (French association for value analysis) is adequate to represent the need addressed by the innovation. This is used for representing the AS-IS, as well as the TO-BE, which may be different from the AS-IS. Each action of one external element upon the system determines a Global Constraint Function (GCF). (See figure 2).

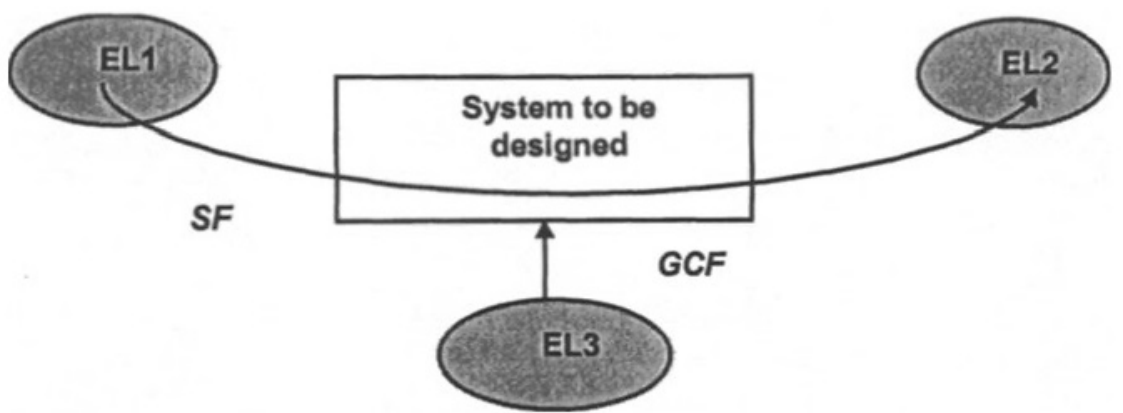

Figure 2

\subsection{The external elements characterisation matrix}

This matrix is used to represent the specific influence of each external element upon the system to be designed. (see figure 3 ).

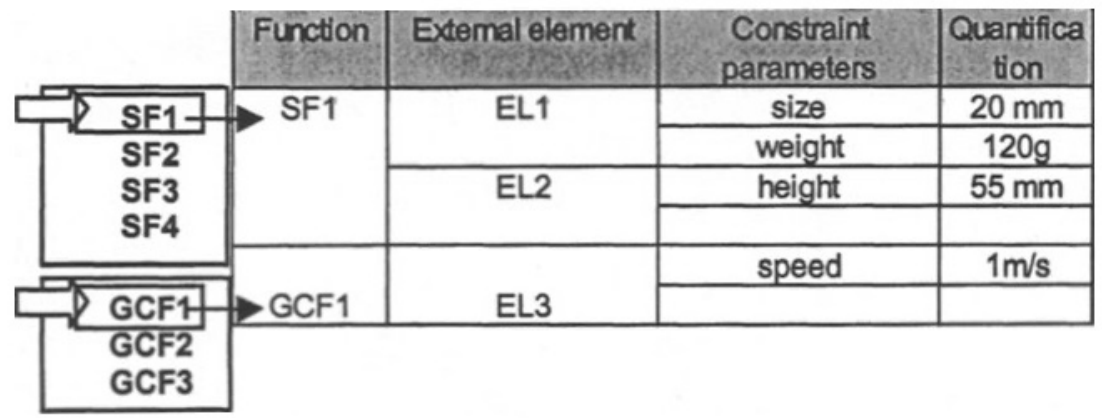

Figure 3

All service functions and global constraint functions are represented in this matrix, and for each, the corresponding external elements are recalled. On the same line, a list of the parameters of the element that constrain the function is detailed, as well as the value of that parameter in the appropriate unit. This matrix has to be filled for the AS-IS and for the TO-BE. 


\subsection{The specification matrix}

The specification matrix is used to characterise each Service Function. The specification is naturally functional (the expected performance of the function). A specific reporting area has been introduced to characterise the economical aspects of the function (acquisition cost, maintenance cost, ..), which are also objectives of the design. The last horizontal is used to perform a projection of each Global Constraint Function upon the considered Service Function. A list of generic Global Function classes, potentially constraining all service functions, can be imagined (legal, safety, quality, aesthetic, ergonomic, environment, ...). Figure 4 shows an example of such specification matrix.

\begin{tabular}{|l|l|}
\hline Feature & \multicolumn{1}{|c|}{$\begin{array}{c}\text { Feature } \\
\text { parameter }\end{array}$} \\
\hline Functional & \\
\hline & cycle time \\
\hline & precision \\
\hline Economical & \\
\hline & acquisition \\
\hline & repair cost \\
\hline Derived from GCF & \\
\hline & reliability \\
\hline
\end{tabular}

\begin{tabular}{|c|c|c|}
\hline Aim & Flexibility & $\begin{array}{c}\text { Measured } \\
\text { value }\end{array}$ \\
\hline $3 \mathrm{~s}$ & low & $2 \mathrm{~s} 78$ \\
\hline $1 \mathrm{~mm}$ & idle & $1 \mathrm{~mm}$ \\
\hline & & \\
\hline$\$ 120$ & high & $\$ 140$ \\
\hline$\$ 35$ & high & $?$ \\
\hline & & \\
\hline $99,99 \%$ & idle & $98 \%$ \\
\hline
\end{tabular}

Figure 4

On the right side of the matrix, a description of the AS-IS (or a TO-BE) situation is given. The aim concerning each feature parameter is indicated by an expected value. A flexibility, according to the designer's opinion, is also indicated for each feature, so as to characterise the relative importance of each feature (a mark or a lexical value can be used indifferently). In the last column, it is interesting to represent, for the considered feature, the value actually measured when available, so as to suggest correcting actions.

\subsection{The criticality analysis matrix}

This matrix is used to synthesise, at the need level, the general perception of a To-BE scenario as perceived by each actor impacted. Depending on the actor's role in the life cycle, the impact can be on the cost, or on the value, or both. The leftmost header of the two previous matrices, where the interesting features of each service function are detailed, are re-considered. Then, for each impacted user identified in step 1 of the method, the criticality of the 
parameter is questioned. The operational manner used to obtain the value is not the purpose of this paper, but we can imagine questionnaires or physical meetings, based on papers or on the partners' Intranet facility, ... An example of such a matrix for a given service function is represented figure 5 .
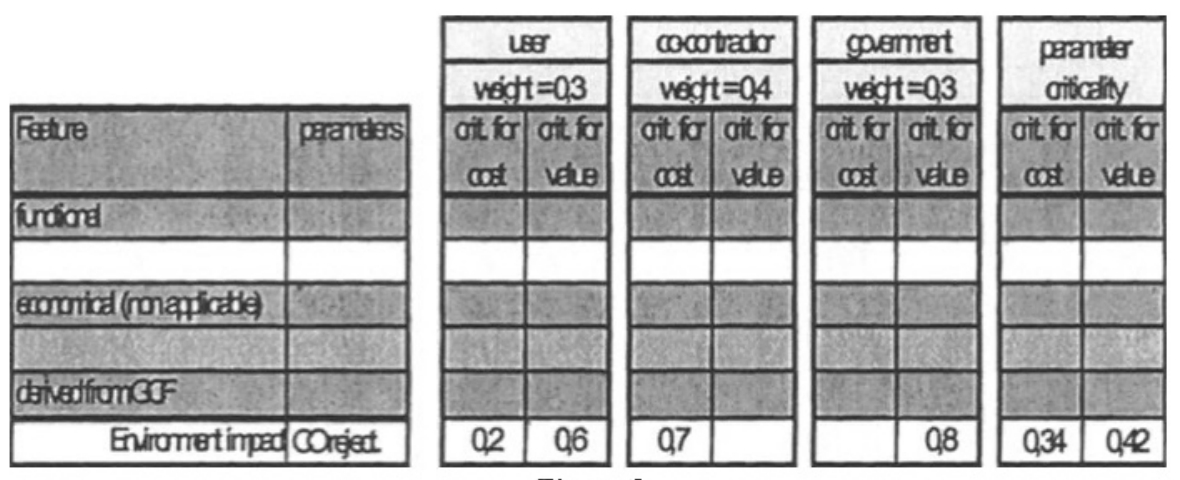

Figure 5

In the above example, a service function implementing a motorised action is addressed. The new feature considered in the TO-BE scenario is a better preservation of the environment, by a reduction of the emission of $\mathrm{CO}$ gas. This feature is not equally perceived by the different actors. The user considers that a low emission is valuable (e.g.: $0.6 / 1$ ). At the same time, he will have to bear an additional cost $(0,2 / 1)$, affecting the acquisition and or the maintenance of this property. The co-contractor responsible for the elaboration of the system gets no added value from that property, but has to modify his production system, which induces a important cost $(0.8 / 1)$. The government, who favours environment friendly systems, will strongly appreciate this feature without supporting any cost. The last column can be used to characterise the overall criticality of each parameter for the cost and the value of the TO-BE scenario, based on the respective opinions of the actors, weighted by the importance of the actor in the project.

\section{CONCLUSION}

Under the combined pressure of the laws, the technological improvements, the market, the competitors, etc,...companies are faced with the necessity to innovate for remaining competitive. A technical innovation concerning an existing product is an economical process, aiming at integrating an invention into an existing product. The success of this process depends on the global cost of that innovation during the whole life-cycle of 
the product, which is determined early in the innovation cycle, and on the balance of the value sharing among the different actors of this life-cycle. So, it is a critical issue to appreciate these features during the assessment phase of the aimed innovation.

This paper has introduced a method for fostering an objective evaluation of the impacts of TO-BE scenarios on the global cost, with a view to permit a comparative analysis of a TO-BE against the AS-IS, or of a TO-BE against other TO-BEs.

The method starts from an identification of the aim of the innovation, which determines the main impacted actors. The own models of the AS-IS situation are then considered for each actor. These models serve as the basis for the representation of the modifications introduced by the TO-BE. This process can be reproduced in any product modelling domain, from the need model to the detailed models. In this paper, only the need models have been explored. The last step of the method consists in synthesising the overall criticality of the innovation, which depends on the individual criticality perceived by each actor. Neither the methodology, nor the instruments for actually collecting the necessary information or for evaluating a TO-BE were addressed in the paper.

The propositions reported in this paper are an embryo of a method elaborated within the scope of an ongoing research project in collaboration with a laboratory of economics and a car's manufacturer. The method is based on the analysis of a recent technical innovation carried out by the industrial partner. The immediate perspectives of this work are: 1) to investigate the models in the functional, technological and technical modelling domains ; 2) to implement these models on a computer thus enhancing their consistency 3 ) to use the method for accompanying an innovation being carried out.

\section{REFERENCES}

Barreyre P.Y. (1981). Typologie des innovations, [typology of innovations]. Revue française de gestion, Jan-Feb issue.

Eschenbächer J., Cocquebert E. (1999). Managing extended enterprise manufacturing networks by using the electronic assistant (GENCMM). IMS'99, Leuwen, Belgium, pp 393-403.

Fernez-Walch S. (1991). L'innovation de produit au quotidien en entreprise industrielle, [Daily product innovation in industrial companies]. Thesis Dissertation, Ecole des Mines, Paris.

Jacquet L. (1998). Contribution à l'élaboration d'une démarche de spécification fonctionnnelle. [Contribution to the development of a functional specification approach] thesis dissertation, University of Valenciennes. 
Kline S., Rosenberg N. (1986).An overview of innovation, Landau R., Rosenberg N. (eds), The positive Sum Strategy, National Academy Press, Washington.

Marciniak R., Pagerie M. (1999). Management de projet : méthodes et outils, [project management: methods and tools], WEKA Ed.

Meredith J., Mantel S. J. Jr (1995). Project Management. A managerial approach, John Wiley \& sons, New York.

NFX50-150 (1983). Record of the AFNOR standard: Analyse de la valeur - vocabulaire. [the vocabulary of value analysis].

Nordlund M., Tate D., Suh N.P. (1996). Growth of Axiomatic Design Through Industrial Practice, Proceedings of the CIRP Workshop on Design and Implementation of Intelligent Manufacturing systems, University of Tokyo.

Renaud J., Boly V., Guidat C. (1999). Etude de la pérennisation du processus d'innovation en entreprise [study about the durable implementation of an innovation process within a company], $3^{\text {rd }}$ international congress of industrial engineering, Montréal, Quebec, May, pp 1673-1681.

Schumpeter J. (1935). Théorie de l'évolution économique, [Theory of Economical Evolution (initial publication in 1912)], French version by Dalloz, Paris.

Vadcard P. (1995) Méthodologie de conception de produit, l'évolution des représentations du produit, [product design methodology, the evolution in product representation]. Proceedings of the CONFERE seminar about the designer's representation tools and technologies, ENSAM, Paris, April.

Xuereb J.-M. (1991). Une redéfinition du processus d'innovation [a new definition of the innovation process] Revue française de gestion, june-august issue. 